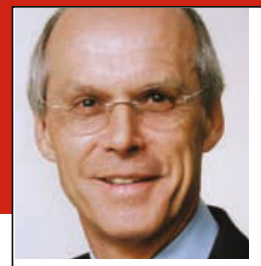

Prof. Dr. med. H. S. FüeßI Isar-AmperKlinikum, KI. MünchenOst, Haar

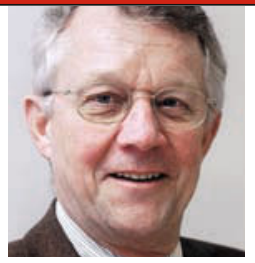

Prof. Dr. med. H. Holzgreve

Internist, Kardiologische

Praxis, München

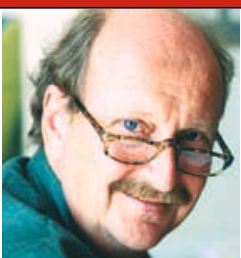

Prof. Dr. med.

E. Ernst

Peninsular

Medical School,

University

of Exeter/UK

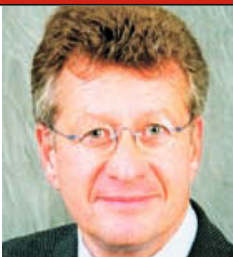

Dr. med. P. Stiefelhagen

Westerwald Krankenhaus, Hachenburg

\title{
Prostatakarzinomvorsorge rettet keine Leben
}

\begin{abstract}
Eine umfangreiche Metaanalyse stützt sich auf neue Studien seit 2005 und kommt zu dem Ergebnis, dass die PSA-Bestimmung zwar Karzinome entdeckt, die anschließende Behandlung aber die Lebenserwartung langfristig nicht verbessert.
\end{abstract}

- Zwischen 2005 und Juli 2010 wurden sechs Studien publiziert. Bei den 387286 asymptomatischen Probanden wurde nach Zufallskriterien entweder das PSA bestimmt oder darauf verzichtet. In beiden Armen wurde operativ behandelt, sobald ein Prostatakarzinom gesichert wurde.

Naturgemäß entdeckte man bei den Probanden mit PSA-Bestimmung sofort oder im weiteren Verlauf signifikant häufiger Prostatakarzinome als ohne Screening (64 vs. 44 pro 1000 Probanden; Zunahme um 46\%; $\mathrm{P}<0,001$; s. Tab.). Dabei handelte es sich vorwiegend um das frühe Stadium I, seltener um die Stadien II bis IV. Entscheidend ist, dass während der Studiendauer zwischen 4 und15 Jahren durch die Vorsorgeuntersuchung und die anschließenden Maßnahmen die prostataspezifische Mortalität nur um 12\%, die Gesamtsterblichkeit nur um 1\% abnahm.

In absoluten Zahlen bedeutet dies, dass durch die Vorsorge pro 1000 Pati-

\begin{tabular}{|l|l|l|}
\hline Tabelle 1 & & \\
\hline $\begin{array}{l}\text { Prostata- } \\
\text { diagnose }\end{array}$ & $+46 \%$ & $\mathrm{~s}(\mathrm{P}<0,001)$ \\
\hline $\begin{array}{l}\text { Gesamt- } \\
\text { mortalität }\end{array}$ & $-1 \%$ & ns $(\mathrm{P}<0,44)$ \\
\hline $\begin{array}{l}\text { Tod infolge } \\
\text { Prostata- } \\
\text { karzinom }\end{array}$ & $-12 \%$ & ns $(\mathrm{P}<0,25)$ \\
\hline
\end{tabular}

Auswirkungen des PSA-Screenings auf Diagnose, Gesamtmortalität und prostataspezifische Mortalität.

enten nur 7 statt 8 am Prostatakarzinom verstarben und die Gesamtsterblichkeit nur von 200 auf 198 zurückging. Diese Ergebnisse verfehlten die Signifikanz deutlich. Die Auswertung für fünf verschiedene Altersgruppen zwischen 50 und 75 Jahren ergab für die relativen krankheitsspezifischen Mortalitätsraten nach Screening Abnahmen von $-10 \%,-27 \%,-6 \%,-26 \%$ und $+26 \%$, d.h. weder eine überzeugende Linearität noch Signifikanzen. Leider konnten mangels Daten die Nebenwirkungen und Folgen von Screening und Operation nicht analysiert werden.

\footnotetext{
- M. Djubegovic et al.

Screening for prostate cander: systematic review and meta-analysis of randomised controlled trials. Brit. Med. J. 341 (2010) 593
}

\section{Kommentar}

Das Prostatakarzinom ist nach dem Lungenkarzinom die zweithäufigste Todesursache durch bösartige Erkrankungen bei Männern. Vorsorgeuntersuchungen sollen krankheitsspezifische Todesfälle verhindern und die Gesamtsterblichkeit verringern. In umfangreichen Studien mit großen Patientenzahlen wurde dieses Ziel nicht erreicht, obwohl durch die Vorsorgeuntersuchung mehr Prostatakarzinome entdeckt werden. Dies kann nur so gedeutet werden, dass viele der entdeckten Karzinome niedrig maligne sind, einen langsamen natürlichen Verlauf haben und innerhalb der Lebenserwartung zu keinen Folgen führen..

Es ist kaum zu erwarten, dass künftige Studien dieses Gesamtbild modifizieren werden, solange es nicht gelingt, durch zuverlässige, z.B. biochemische Kriterien die Aggressivität der Tumoren besser zu prognostizieren und die Behandlung auf hochmaligne Tumoren zu begrenzen. Die Leitlinien der Deutschen Gesellschaft für Urologie stellen fest, dass eine Lebensverlängerung durch PSA-Screening nicht belegt ist. PSA und rektale Untersuchung sollten nur Männern mit Wunsch nach einer Früherkennung empfohlen werden. Aber wer sollte den Wunsch wecken?

H. HolzGREVE 